\title{
Investigation the Role of Autophagy in Non-Small Cell Lung Cancer
}

\author{
Minoo Pargol ${ }^{1,2}$, Shima Zare Karizi ${ }^{1,2}$, Masoumeh Akbari ${ }^{1,2}$, Bahareh \\ Nourmohammadi ${ }^{2}$, Mohammad Behgam Shadmehr ${ }^{3}$, Morteza Karimipoor ${ }^{2 *}$ \\ Shohreh Zare Karizi ${ }^{1 *}$
}

\begin{abstract}
Objective: Recent studies have shown the role of autophagy in different types of cancer including lung cancer. MicroRNAs are considered as key factors in regulation of autophagy related genes. miR-30d, miR-204-5p and miR-20a are regulatory markers which can suppress the expression of beclin1, LC3, bcl2 and ULK1 as their target genes and they lead to decrement of autophagy in human cancer cells. Moreover, epigenetic modifications DNA methylation has been indicated in regulation of autophagy in different stages of cancer. Methods: In this study, the expression levels of miR-30d, miR-204-5p and miR-20a as well as their target genes were analyzed in 30 non-small cell lung cancers (NSCLCs) patients sample and adjacent normal tissues by real-time qPCR. In addition, DNA methylation of beclin1, LC3, bcl2 and ULK1 genes were assessed by MS-HRM method. Results: MiR-30d ( $p$ value $=0.01)$ and miR-204-5p $(\mathrm{P}=0.048)$ significantly down-regulated in tumor samples compared to normal adjacent tissues, while there was no significant change in expression level of miR-20a. On the other hand, target genes expression level was significantly increased in NSCLC tissues, however methylation pattern of the target gene promoters, did not show any significant alteration. Conclusion: These results indicate roles for miR-30d, miR-204-5p as tumor suppressor genes as well as target genes as oncogenes in NSCLC patients. Although these factors may have a significant role in NSCLC progression, further studies are necessary to investigate the implications of these findings for treatment of lung cancer.
\end{abstract}

Keywords: MicroRNAs- non-small cell lung cancer- autophagy- DNA methylation

Asian Pac J Cancer Prev, 22 (3), 947-955

\section{Introduction}

Lung cancer is the leading cause of cancer related death worldwide. Non-small cell lung cancer (NSCLC) accounts for approximately $85 \%$ of all lung cancer cases. NSCLC is histologically classified to three main subtypes: squamous cell carcinoma (SCC), large cell carcinoma (LCC) and adenocarcinoma (ADC) (Hou et al., 2010). The last two subtypes, compromises $25-30 \%$ and $40 \%$ of all NSCLC patients, respectively. The prognosis of lung cancer remains poor and despite various progresses in diagnostic and therapeutic processes, the 5-year survival has only increased from $15.7 \%$ to $17.4 \%$ during the last decade (Boolell et al., 2015).

Macro-autophagy (hereafter referred to as autophagy), which is a homeostatic mechanism regulating degradation of proteins, organelles, and other cellular components in lysosomes, plays an important role in cancer initiation, progression and development (Frankel and Lund, 2012). It is an evolutionary conserved phenomenon observed in all eukaryotic cells, from yeast to mammals involving in turnover of proteins and organelles as well as adaptation and survival, or death in different environmental unfavorable conditions such as nutrient deprivation and pathogen infection (Kondo et al., 2005). Therefore, malfunction of this catabolic pathway contributes to various human diseases including cancer (Frankel et al., 2011).

Approximately 30 different genes have been studied to be involved in regulating autophagy (Wang et al., 2015). Beclin 1, the mammalian orthologue of yeast Atg6, participates in autophagosome formation by interacting with type III phosphatidylinositol 3-kinase (PI(3)KC3) complex (Takahashi et al., 2007). Beclin1 possesses a conserved domain (ECD), mediating its interaction with $\mathrm{PI}(3) \mathrm{KC} 3-\mathrm{Vps} 34$, as well as bcl-2-homology-3

${ }^{1}$ Department of Genetics and Biotechnology, School of Biological Science, Varamin Pishva Branch, Islamic Azad University, Varamin, Iran. ${ }^{2}$ Molecular Medicine Department, Biotechnology Research center, Pasteur Institute of Iran, Tehran, Iran. ${ }^{3}$ Tracheal Diseases Research Center, National Research Institute of Tuberculosis and Lung Diseases Shahid Beheshti University of Medical Sciences, Tehran,Iran.*For Correspondence: mortezakarimi@pasteur.ac.ir, shohrehzare@yahoo.com. Minoo Pargol, Shima Zare Karizi and Masoumeh Akbari have equal contribution in this study. 
(BH3) domain that regulates its binding to bcl-2 family (particularly bcl-2 and its homologue Bcl-XL) (Maiuri et al., 2007). Bcl-2 (B-cell lymphoma/leukemia-2), the antiapoptotic protein, suppresses the autophagic activity of the Beclin1-PI(3)KC3 complex via its binding to Beclin1 during non-starvation conditions (He and Klionsky, 2009). Microtubule associated protein light chain 3 (LC3), the mammalian counter part of yeast Atg8, also plays a pivotal role in autophagosome formation (Jiang et al., 2012). LC3 includes soluble and membrane-bound forms, LC3 I and LC3 II respectively (Miracco et al., 2010). Various types of stressors induce the conversion of LC3-I to LC3-II, which has been considered to be the most reliable autophagosomal marker (Jiang et al., 2012). UNC-51-like kinase 1 (ULK1), the homologue of Atg1 in mammals, is a multifunctional protein that regulates initial events of autophagy signaling (Chen et al., 2014).

MicroRNAs (miRNAs) are small non-coding RNA molecules that regulate the expression of their target genes at post-transcriptional level. They have essential roles in controlling various cellular activities including differentiation, proliferation, apoptosis and tumorigenesis. Several studies indicate that miRNAs are also involved in the regulation of autophagy. Inhibition of some autophagic proteins by miRNAs has been suggested to hinder the progression of lung cancer (Liu and Huang, 2015). $m i R-30 \mathrm{~d}$, member of mir-30 miRNA family, regulates numerous genes in autophagy process including beclin1. It has been shown that miR-30d suppresses the expression of beclin1 gene, which leads to decreasing autophagy in human cancer cells (Yang et al., 2013; Zhao et al., 2017a). MiR-204-5p (previously named as miR-204 before miRBase release 19.0) shows significantly reduced expression and exerts its function as a tumor suppressor in several cancers such as glioblastoma, prostate cancer and NSCLC (Luan et al., 2017). It has also been determined miR-204-5p is related to autophagy pathway in various types of cancers and increased function of LC3-II in autophagy is inhibited by increasing miR-204-5p level in colorectal cancer (Sümbül et al., 2014). Bcl-2 is also the functional target of $m i R-204-5 p$, which decreases its protein expression by targeting 3'-untranslated region (UTR) of bcl-2 (Sacconi et al., 2012). MiR-20a is a member of the miR-17-92 cluster, which has been widely studied to have oncogenic function. MiR-20a has been shown to regulate autophagy pathway negatively in breast cancer cells. Moreover, inhibition of leucine deprivation-induced autophagy via down-regulation of ULK1 by miR-20a has been demonstrated in C2C12 myoblasts (Guo et al., 2016).

DNA methylation is a significant epigenetic mechanism that happens by addition of a methyl group to the cytosine, mostly on $\mathrm{CpG}$ islands in promoter region, contributes to the silencing of gene transcription (Xu et al., 2018). Moreover, epigenetic modifications, including silencing of various significant genes by DNA methylation, have been demonstrated in regulation of autophagy in different stages of cancer. Aberrant promoter methylation of beclin 1 gene leads to downregulation of its protein in breast cancer tissues. Methylation condition of autophagic genes may play fundamental role in cancer progression. Therefore, understanding novel epigenetic functions in autophagy regulation may contribute to outstretch strategies for cancer diagnosis and treatment (Sui et al., 2015).

The aim of present study was to evaluate the methylation and expression levels of genes involved in autophagy process and the related miRNAs in NSCLC samples.

\section{Materials and Methods}

In this study, 30 specimens from tumoral tissues and 30 specimens from the adjacent normal tissues were taken from the resected lung lobes in patients who underwent curative surgical resection (lobectomy) for NSCLC from 2011 to 2014 at Masih Daneshvari Hospital (Tehran, Iran). The patients did not receive any preoperative chemotherapy or radiotherapy.

The pathological characteristics of all samples were obtained according to standard protocols from pathologists. The clinico-pathological features of the patients are summarized at Table 1. The study was approved by the ethics committee (sbmu 1. REC.1390. 65) of the Masih Daneshvari Hospital and written informed consent was taken from all patients before surgical resection. Fresh tissue samples were transferred immediately to liquid nitrogen and stored at $-80^{\circ} \mathrm{C}$ until use.

\section{RNA extraction and $c D N A$ synthesis}

Total RNA was extracted from tumoral and adjacent normal tissues by using Tripure Isolation Reagent (Qiagen, USA) according to the manufacturer's instruction. The purity and concentration of extracted RNA was measured by Nanophotometer. For cDNA synthesis, mature miRNA was reverse transcribed using specific stemloop primers (Reverted First Strand cDNA Synthesis Kit, Thermo, Lithuania). In brief, 1,000 ng RNA of each sample, 15 pmol stem loop primer, $4 \mu \mathrm{l} 5 \mathrm{x}$ buffer, $2 \mu \mathrm{dNTP}$, $0.5 \mu$ Ribo Lock RNAse inhibitor and $0.5 \mu$ reverse transcriptase enzyme were added in total volume of 20 $\mu \mathrm{l}$. The thermal cycling condition for the cDNA synthesis was as follows: $42^{\circ} \mathrm{C}$ for $60 \mathrm{~min}$ followed by $70^{\circ} \mathrm{C}$ for 5 min. For cDNA synthesis of target genes, total RNA was reversely transcribed into cDNA using Random hexamer primers (RevertAid First Strand cDNA Synthesis Kit, Thermo, Lithuania). In summary, 1,000 ng RNA of each sample, 10 pmol Random hexamer primers, $4 \mu 15 \mathrm{x}$ buffer, $0.5 \mu$ Ribo Lock RNAse inhibitor, $2 \mu \mathrm{dNTP}$, and $0.5 \mu l$ reverse transcriptase enzyme were added in the total volume of $20 \mu \mathrm{l}$. The thermal cycling condition for the cDNA synthesis was as follows: $25^{\circ} \mathrm{C}$ for $5 \mathrm{~min}$ and $42^{\circ} \mathrm{C}$ for $60 \mathrm{~min}$ followed by $70^{\circ} \mathrm{C}$ for $5 \mathrm{~min}$.

\section{Primer design and $q R T-P C R$}

Gene-specific primers for target genes and also forward and universal reverse primers for miRNAs were designed using GeneRunner and Allele ID 6.0 softwares (Tables 2 and 3). In this study, for detection of miRNA expression level as well as target genes in tumor and adjacent normal tissues, SYBER qRT-PCR was performed. All steps were implemented according to the protocols and all samples were performed in duplicate in MicroAmp optical 
96-well plate (StepOne plus Applied Biosystem USA). The reactions were normalized with RNU44 control for miRNAs and GAPDH for target genes. In brief, for detection of expression pattern of $m i R-20 a, m i R-30 d$ and $m i R-204-5 p$ each reaction contains $10 \mu \mathrm{L}$ SYBR master mix, 5 pmol of specific forward and universal reverse primers and $2 \mu \mathrm{l}$ of cDNA in total volume of $20 \mu \mathrm{l}$ reaction. The thermal cycling condition for the amplification of mentioned miRNAs were as follows: initial denaturation at $95^{\circ} \mathrm{C}$ for $15 \mathrm{~min}$, followed by 40 cycles of $95^{\circ} \mathrm{C}$ for 20 $\mathrm{s}$ and $60^{\circ} \mathrm{C}$ for $1 \mathrm{~min}$ for miR-20a and miR-204-5p as well as initial denaturation at $95^{\circ} \mathrm{C}$ for $15 \mathrm{~min}$, followed by 40 cycles of $95^{\circ} \mathrm{C}$ for $20 \mathrm{~s}$ and $62^{\circ} \mathrm{C}$ for $1 \mathrm{~min}$ for miR-30d. In addition, expression level of Beclin-1, bcl2, ULK1 and $L C 3$ was measured by SYBER qRT-PCR. Each reaction contains $10 \mu \mathrm{S} \mathrm{SYBR}$ master mix, 5 pmol of specific forward and reverse primers and $400 \mathrm{ng}$ of cDNA in total volume of $20 \mu \mathrm{l}$ reaction. The samples were analyzed in duplicates and performed in MicroAmp optical 96-well plate. GAPDH was used as a reference gene using ABI software (StepOne plus, Applied Biosystems). The thermal cycling conditions were as follows: initial denaturation at $95^{\circ} \mathrm{C}$ for $15 \mathrm{~min}$, followed by 40 cycles of $95^{\circ} \mathrm{C}$ for $15-30 \mathrm{~s}$ and $60^{\circ} \mathrm{C}$ for $1 \mathrm{~min}$. Moreover, the efficiency of amplification for each miRNA and target gene was assessed by serial dilution of cDNA.

DNA methylation of Beclin-1, bcl2, LC3 and ULK1

Methylation specific- high resolution melting (MSHRM) method was exploited for methylation analysis. Gene-specific primers for CpG islands of Beclin-1, bcl2, $L C 3$ and $U L K 1$ genes were designed using Generunner and Zymoresearch softwares (Table 4). DNA from NSCLC and paired normal adjacent tissues was extracted using QIAamp DNA extraction kit (Qiagen, United States). Then DNA was quantified and used for bisulfite conversion with the EZ DNA Methylation Gold (Zymo research, USA) in order to perform DNA methylation of Beclin-1, bcl2, LC3 and ULK1 genes. The thermal cycling conditions were as follows: preliminary denaturation at $98^{\circ} \mathrm{C}$ for $10 \mathrm{~min}$ followed by $64^{\circ} \mathrm{C}$ for 2.5 hours and then stored at $4^{\circ} \mathrm{C}$ up to 20 hours. In addition M.SssI enzyme (Thermo Fisher Scientific, USA) was used to produce fully methylated DNA control. This bisulfite converted DNA was then used for the MS-HRM assay. In brief, $4 \mu \mathrm{l} 5 \mathrm{x}$ HOT FIREPol EvaGreen HRM Mix, 6 pmol forward and reverse primers and $1 \mu \mathrm{l}$ of bisulfite-treated DNA in total volume of $20 \mu \mathrm{l}$ reaction was performed.

\section{Data collection and statistical analysis}

The expression level of miRNA and target genes were measured by using the comparative $2^{-\triangle \Delta C T}$ (fold change) method. The statistical significance of relative changes in $m i R N A$ and $m R N A$ expression between different groups of lung tumors were determined by t-test. P-value of $<0.05$ was considered as statistically significant. The graphs were created by GraphPad PRISM 5.0 software. According to statistical analysis for DNA methylation, Fisher's exact test and IBM SPSS Statistics 21 software was determined by t-test.

\section{Results}

Expression level of $m i R-20 a, m i R-30 d$ and $m i R-204-5 p$ and target genes in tissue samples

In this study, we measured the expression level of $m i R-20 a, m i R-30 d$ and $m i R-204-5 p$ as well as their target
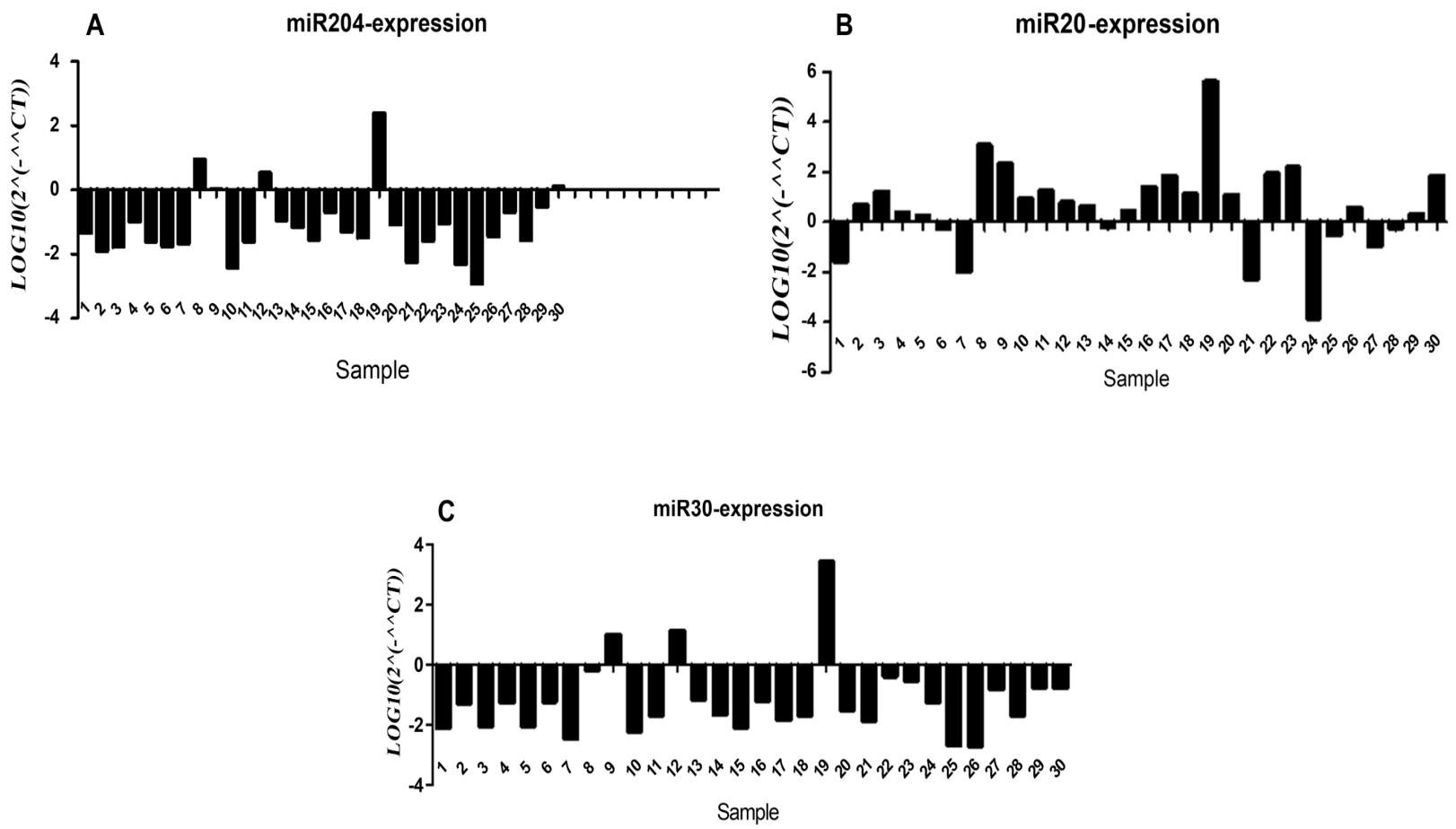

Figure 1. The Expression Level of miR-204-5p (A), miR-20a (B), and miR-30d (C) in NSCLC Patients Compared to Normal Adjacent Tissue. $m i R-204-5 p$ expression was significantly decreased $(\mathrm{P}=0.048)$. No significant was found in the expression of $m i R-20 a(\mathrm{P}=0.5)$. miR-30d expression level in NSCLC patients compared to normal adjacent tissue was significantly decreased $(\mathrm{P}=0.01)$. 


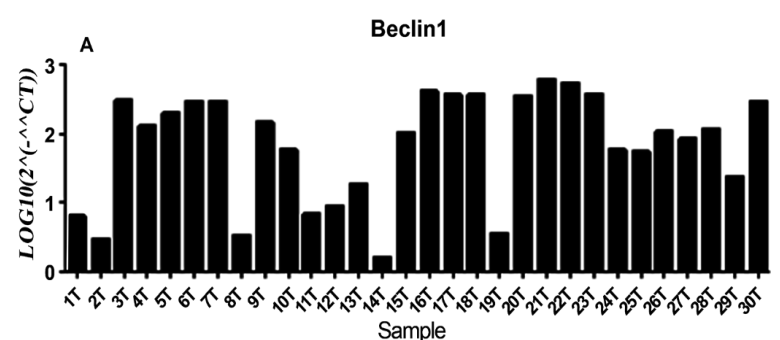

Sample

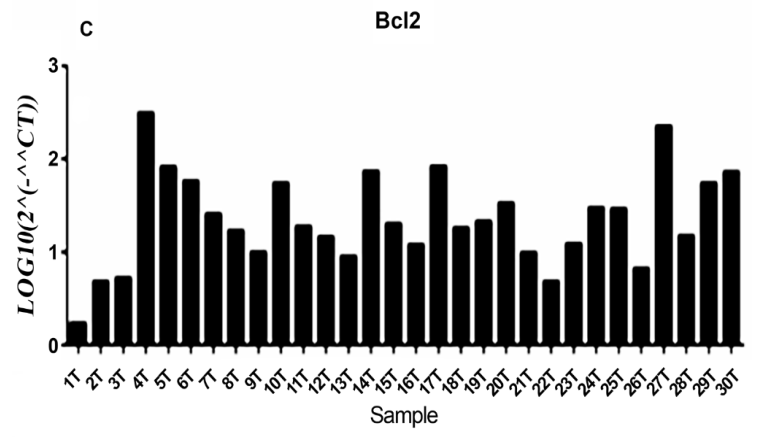

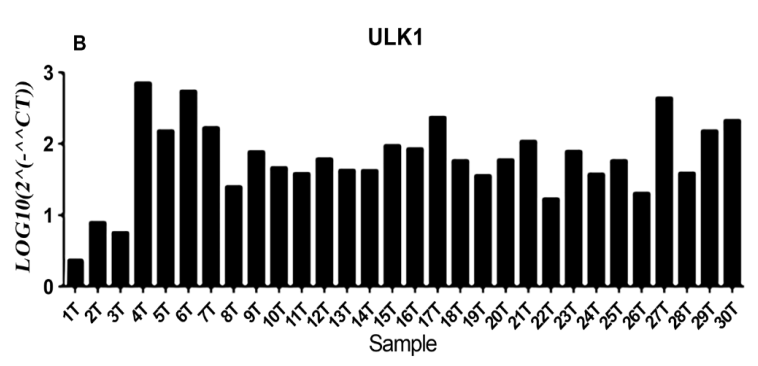

D

LC3

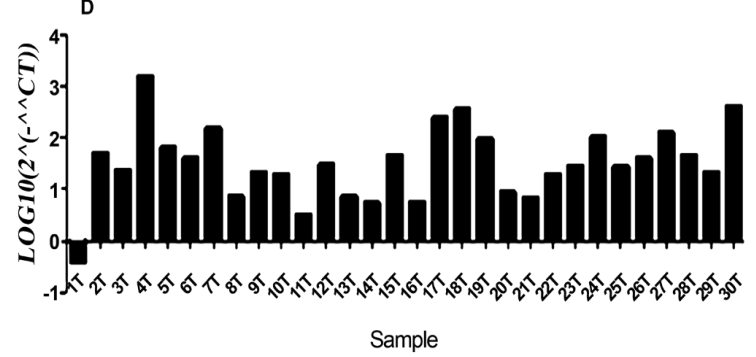

Figure 2. The Expression Level of Beclin1, ULK, bcl2, and LC3 in NSCLC Patients Compared to Normal Adjacent Tissue. (A) Beclin1 expression was significantly increased in NSCLC samples compared to normal adjacent tissues (p-value $<0.0001$ ). (B) up-regulation of $U L K 1$ was found in patients with NSCLC compared to normal adjacent tissue. ( $\mathrm{p}$-value $<0.0001$ ). (C) bcl2 expression was significantly increased in NSCLC samples compared to normal adjacent tissues (p-value $<0.0001$ ). (D) LC3 expression was significantly increased in NSCLC samples compared to normal adjacent tissue ( $\mathrm{p}$-value $<0.0001)$.

genes (Beclin1, ULK1, bcl2 and LC3) in 30 Iranian patients with NSCLC tumoral samples compared to normal adjacent tissues. The target genes were selected by Targetscan and miRwalk databases according to their high score and more complementary base pairing between miRNAs seed region and 3'UTR sequence of mRNA

Table 1. The Clinico- Pathological Features of NSCLC Patients TNM, Tumor-Node-Metastasis

\begin{tabular}{lc}
\hline Tissue & Case \\
\hline Age & \\
$\quad<60$ & 14 \\
$\geq 60$ & 16 \\
Sex & \\
$\quad$ Male & 23 \\
Female & 7 \\
TNM & \\
I or II & 20 \\
III & 10 \\
Subtype & \\
Adenocarcinoma & 18 \\
SCC & 12 \\
Pack/year & \\
- & 21 \\
4-120 & 9 \\
Lymph node metastasis & \\
Yes & 13 \\
No & 17 \\
\hline
\end{tabular}

of target genes. ULK1 was assigned as miR-20a target gene and Beclin1 was chosen as miR-30d target gene, while $b c l 2$ as well as $L C 3$ were selected as $m i R-204-5 p$ target genes. According to our data obtained in this study, downregulation of miR-30d $(\mathrm{P}=0.01$, Figure $1 \mathrm{C})$ and miR-204-5p $(\mathrm{P}=0.048$, Figure 1A) have been detected in NSCLC patients compared to normal adjacent tissues, while the expression level of miR-20a has not been changed significantly $(\mathrm{P}=0.5$, Figure $1 \mathrm{~B})$. Moreover, we found that the expression level of Beclin1, ULK1, bcl2 and $L C 3$ were significantly increased in NSCLC patients $(\mathrm{P}<0.0001$, Figure 2$)$.

Correlation of miRNAs expression level with clinical and pathological features in NSCLC patients

In this study, the expression level of $m i R-20 a$,

Table 2. Primer Sequences for Expression Analysis of Target Genes

\begin{tabular}{|c|c|c|c|}
\hline \multirow{2}{*}{$\begin{array}{l}\text { Ref Seq Accession } \\
\text { NM_003766.4 }\end{array}$} & \multicolumn{2}{|c|}{ Gene Target } & \multirow{2}{*}{$\frac{\text { Product size }(\mathrm{bp})}{190}$} \\
\hline & Beclin-1 & Forward & \\
\hline & & Reverse & \\
\hline \multirow[t]{2}{*}{ NM_000633.2 } & $b c l 2$ & Forward & 136 \\
\hline & & Reverse & \\
\hline \multirow[t]{2}{*}{ NM_003565.2 } & $U L K 1$ & Forward & 136 \\
\hline & & Reverse & \\
\hline \multirow[t]{2}{*}{ NM_181509.2 } & $L C 3$ & Forward & 193 \\
\hline & & Reverse & \\
\hline \multirow[t]{2}{*}{ NM_001256799.2 } & GAPDH & Forward & 112 \\
\hline & & Reverse & \\
\hline
\end{tabular}



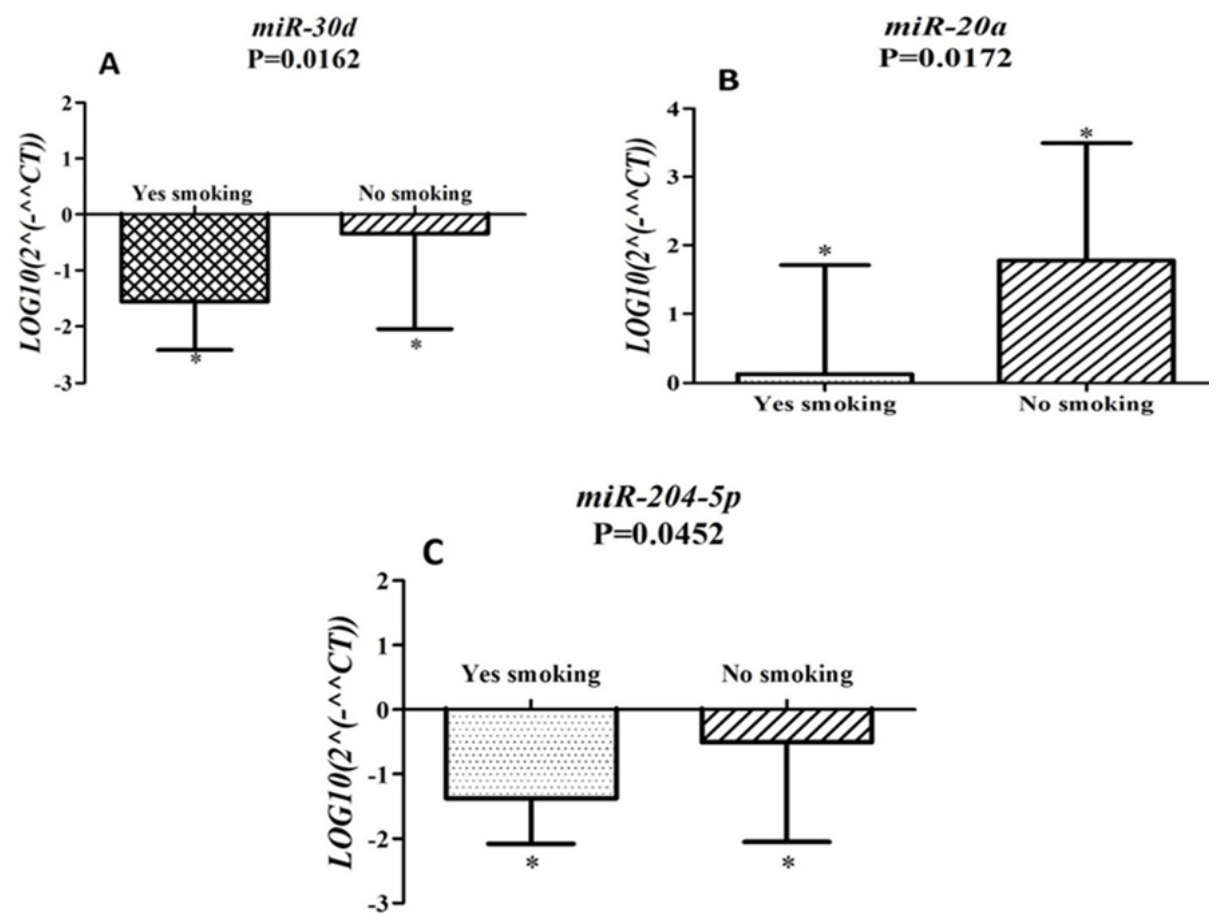

Figure 3. (A) Correlation of miR-30d expression level and smoking. Downregulation of miR-30d expression is correlated with smoking $(\mathrm{P}=0.0162)$. (B) Correlation of miR-20a expression level and smoking. Up-regulation of miR-20a is associated with smoking $(\mathrm{P}=0.0172)$. (C) Correlation of miR-204-5p expression pattern and smoking. Downregulation of miR-204-5p expression is correlated with smoking $(\mathrm{P}=0.0452$

miR-30d and miR-204-5p were compared with subtype (adenocarcinoma and SCC), stage, lymph node involvement and smoking. According to data analyzed, the correlation between overexpression of $m i R-20 a$ $(\mathrm{P}=0.0172$, Figure $3 \mathrm{~B})$ with smoking was detected. Moreover, we found the correlation between downregulation of miR-30d $(\mathrm{P}=0.0162$, Figure $3 \mathrm{~A})$ and miR-204-5p ( $\mathrm{P}=0.0452$, Figure.3C) with smoking. According to our data, expression level of these miRNAs with other clinical and pathological features did not show significant correlation.

Correlation of target genes expression level with clinical and pathological features in NSCLC patients

In our experiment, we analyzed correlation between the expression pattern of Beclin1, ULK1, bcl2 and LC3 with clinical and pathological features (subtype, stage, lymph node metastasis and smoking.). According to data analyzed, overexpression of $L C 3$ ( $\mathrm{P}=0.0171$, Figure.4A) was detected in adenocarcinoma subtype compared to SCC subtype, although the overexpression of bcl2 in adenocarcinoma compared with SCC subtype was less consistent $(\mathrm{P}=0.446$, Figure $4 \mathrm{~B})$. According to our data, expression level of these genes with other clinical and pathological features did not show any significant correlation.

Beclin-1, bcl2, LC3 and ULK1 methylation patterns in NSCLC patients

Difference in methylation levels of Beclin-1, bcl2,

Table 3. Primer Sequences and Stemloops for Quantification of miRNAs

\begin{tabular}{ll}
\hline Primer Name & Primer sequence \\
\hline miR-30d-F & 5' GCGTCCCTGTAAACATC 3' \\
miR-30d-R & 5' GTATCCAGAGCAGGGTCC 3' \\
miR-30d Stemloop & 5'GGTCGTATGCAGAGCAGGGTCCGAGGTATCCATCGCACGCATCGCACTGCATACGCTTCCA3' \\
miR-20a-F & 5'GCGTCCCTAAAGTGCTTATAG3' \\
miR-20a-R & 5'GTATCCAGAGCAGGGTCC3' \\
miR-20a Stemloop & 5'GGTCGTATGCAGAGCAGGGTCCGAGGTATCCATCGCACGCATCGCACTGCATACGCTACCT3' \\
miR-204-5p-F & 5' CTTCCCTTTGTCTTCCTATGC 3' \\
miR-204-5p-R & 5'GTATCCAGAGCAGGGTCC 3' \\
miR-204-5p Stemloop & 5'GGTCGTATGCAGAGCAGGGTCCGAGGTATCCATCGCACGCATCGCACTGCATACGAGGCAT3' \\
RNU44-F & 5' CCT GGA TGA TGA TAG CAA ATG 3' \\
RNU44-R & 5' TCG TAT CCA GTG CAG GG 3' \\
RNU44 Stemloop & 5'GTCGTATCCAGTGCAGGGTCCGACCGGTATTCGCACTGGATACGACAGTCAG3' \\
\hline
\end{tabular}



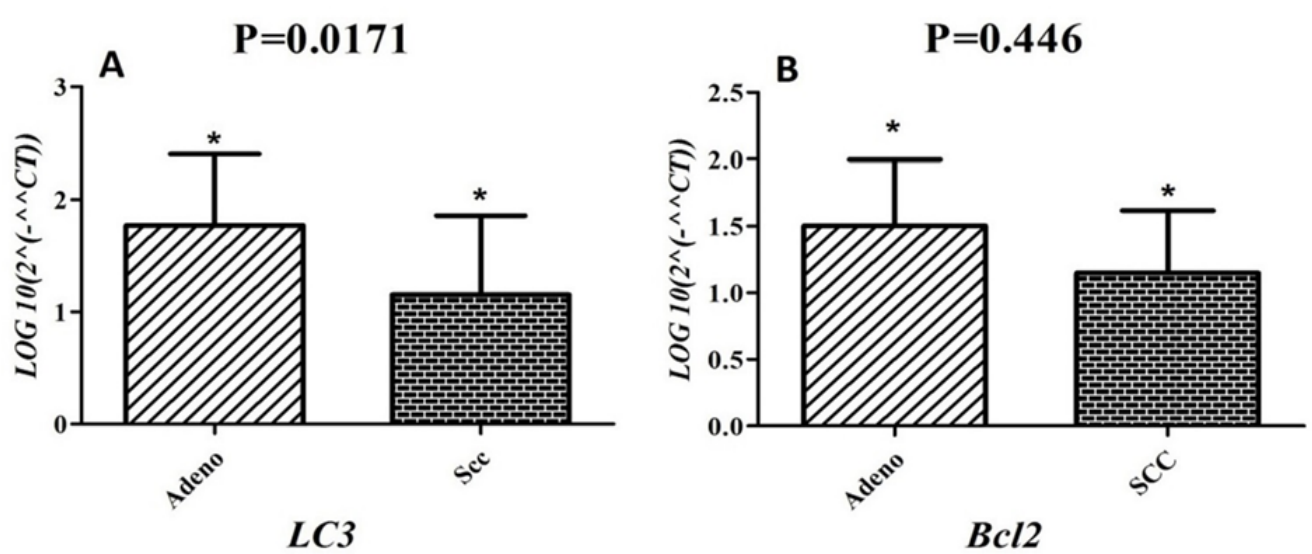

Figure 4. (A) LC3 expression was increased in adenocarcinoma subtype compared to squamous cell subtype (SCC) $(\mathrm{p}=0.0171)$. (B) Up-regulation of $b c l 2$ expression in adenocarcinoma subtype compared to squamous cell subtype (SCC) patients $(\mathrm{p}=0.446)$.

Table 4. Primer Sequences for DNA Methylation Assessment of Genes Related to Autophagy

\begin{tabular}{ll}
\hline Primer Name & Primer sequence \\
\hline Beclin-1- F & 5' GTGAGTTTGTGGATTAGGAGTTTTG 3' \\
Beclin-1-R & 5'CGAAACGAAACCTCCAAAACTAC 3' \\
bcl2-F & 5' GGTCGTGGTAGGTTTGGAAATT 3' \\
bcl2-R & 5' AACTCCGAACAACGCCAAATA 3' \\
LC3-F & 5' CACGAACGCCTATCTCTACAA 3' \\
LC3-R & 5' GATGTCGGGGTAGTAAGTGAT 3' \\
ULK-1-F & 5' TCGTGTGTTTGTTGATATTGTTTT 3' \\
ULK-1-R & 5'CCTCGACCCTAACTACCAA3' \\
\hline
\end{tabular}

A

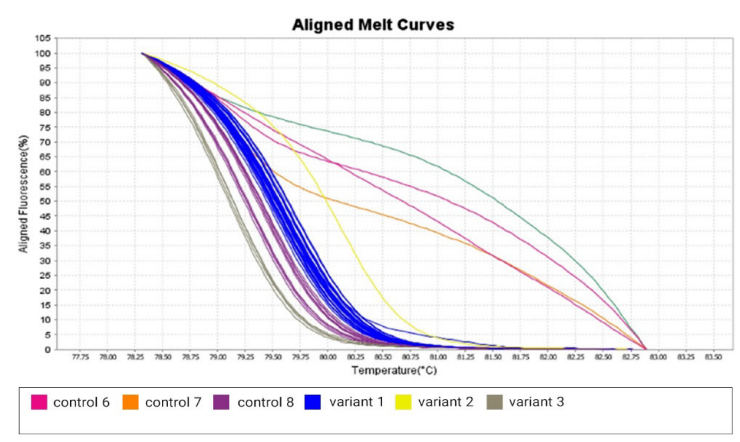

C

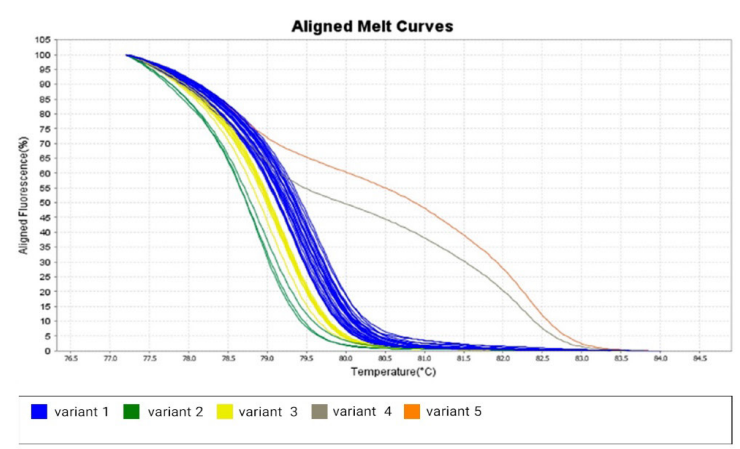

$L C 3$ and $U L K 1$ genes in tumor samples compared to normal tissues were analyzed. According to data obtained, methylation rate of $0-12.5 \%$ in LC3 and ULK1 promoters as well as $0-25 \%$ in bcl2 promoter was detected (Figure.5), however by IBM SPSS Statistics 21 and Fisher's exact test, there is no significant correlation in methylation pattern of these genes in NSCLC samples and normal tissues. Regarding to the data analysis, Beclin-1 promoter was unmethylated in NSCLC tissues (Figure.5) and there is no significant correlation in NSCLC samples compared to normal tissues. In addition, a significant correlation was detected in methylation pattern of LC 3 and bcl 2 promoters

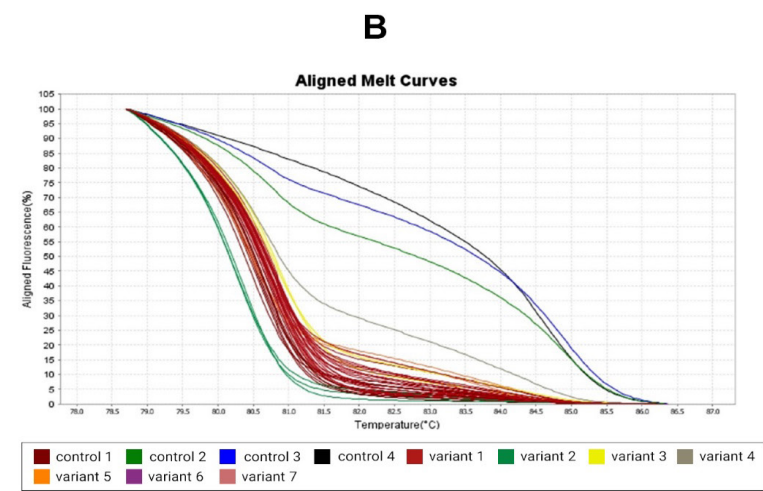

D

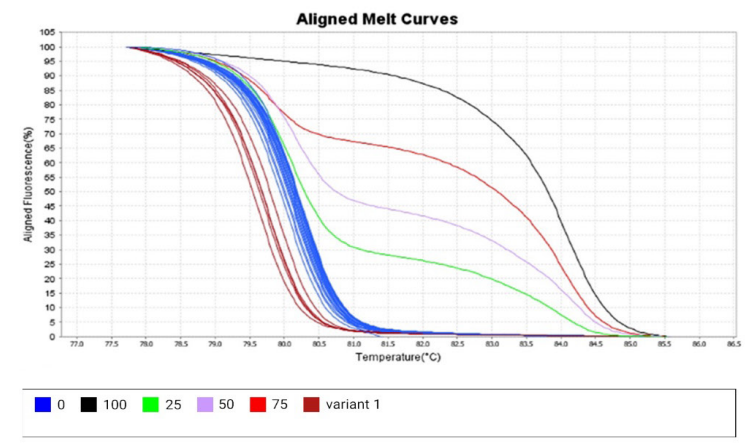

Figure 5. LC3 and ULK1 Promoters were Methylated in 0-12.5\% of NSCLC Samples (A \& B). 0-25\% of methylation was detected in $b c l 2$ promoter of NSCLC samples (C). Beclin_l promoter was unmethylated in NSCLC samples (D). 
by Spearman's Rho test.

\section{Discussion}

Autophagy as a conserved pathway contributes in cellular hemostasis and has a fundamental role in physiologic and pathologic mechanism including cancer. In recent years the role miRNA genes in autophagy regulation has been proposed. As around 90\% of protein-coding genes are regulated by miRNAs, these elements have an important role in the regulation of various physiologic and pathogenic cellular processes including tumorigenesis and invasion (Hannafon et al., 2019). In this study, the expression pattern of $m i R-20 a, m i R-30 d$ and $m i R-204-5 p$ as well as their target genes (Beclin1, $U L K 1, b c l 2$ and LC3) in NSCLC tumoral cells compared with normal adjacent tissues was investigated. According to our data, $m i R-30 d$ and $m i R-204-5 p$ expression were significantly decreased in NSCLC patients, however changes in the expression pattern of $m i R-20 a$ in NSCLC samples compared to normal tissues has not been significant. In addition, the expression level of Beclin1, $U L K 1, b c l 2$ and $L C 3$ were significantly increased in NSCLC patients. These results may show a significant role of miR-30d and miR-204-5p in the development of NSCLC. According to some previous studies, miR-30d is found to be capable of suppressing NSCLC cell growth (Tang et al., 2019), indeed this miRNA can act as a tumor suppressor in NSCLC. Moreover, it has been demonstrated that miR-30d expression increased in hepatocellular carcinoma (HCC), and its expression is highly associated with the intrahepatic metastasis of HCC (Yao et al., 2010). Therefore, $m i R-30 d$ can have a dual function as a tumor suppressor and oncogene in different kind of cancers. According to some studies, miR-204 expression was decreased in NSCLC tumoral samples compared with noncancerous tissue-derived controls. Moreover, miR-204 silencing in NSCLC cell lines promoted proliferation and cell invasion. Thus, this microRNA may be involved in the NSCLC development (Xia et al., 2014). In addition, it has been indicated that $m i R-204-5 p$ expression was down-regulated in cancerous hepatocellular carcinoma (HCC) tissues compared to adjacent normal tissues and Kaplan-Meier survival curve analysis also demonstrated that down-regulation of $m i R-204-5 p$ predicted worse outcomes of HCC patients. Moreover, it has been showed that ectopic expression of $m i R-204-5 p$ in HCC cell lines inhibited cell proliferation, while the prohibition of miR-204-5p enhanced proliferation (Chu et al., 2018). Recent preliminary studies reported that miR-204-5p was inhibited in colorectal cancer and miR-204-5p can decrease cell proliferation and chemo resistance of colorectal cancer cells (Bian et al., 2016). Thus, miR-204-5p act as a tumor suppressor in different kinds of cancers.

In this study, the expression level of Beclin1, ULK1, $b c l 2$ and $L C 3$ were significantly increased in NSCLC patients compared to normal tissues. Recent studies indicated that the expression of Beclin1 and mTOR were well correlated with survival and clinical stages of human non-small cell lung cancer (NSCLC) patients. Indeed, IL-7 activates PI3 K/Akt/mTOR signaling pathway via
Beclin1 to regulate autophagy in lung cancer cells (Jian et al., 2019). What is more, it has been reported that Beclin1 was up-regulated in $67.7 \%$ of NSCLC patients, and was suggested that high Beclin-1 expression predicts longer survival in locally advanced NSCLC (Lee et al., 2019). Thus, this gene may act as an oncogene which is related to longer survival in patients with NSCLC. In our study, ULK1 was significantly over expressed in NSCLC samples. In a recent study was demonstrated that down-regulation of Ulk1 suppresses NSCLC cell growth and sensitizes NSCLC cells to cisplatin by modulating both apoptosis and autophagy pathways (Tang et al., 2017). In addition, it has been indicated miR 21 leads to autophagy related AMPK/ULK1 signaling pathway activation in NSCLC cell lines, while over expression of ULK1 reversed the biological functions of miR 21 . Indeed, miR 21 regulates autophagy activity by AMPK/ ULK1 signaling pathway, and increases the proliferation, migration and invasion of NSCLC cells (Li et al., 2018). According to these data, ULK1 may act as an oncogene and tumor suppressor gene and this factor might be a promising target for NSCLC treatment. Bcl2 and LC3 are two more factors which were analyzed in this study. According to the data obtained, the overexpression of these markers was found in NSCLC patients compared to normal samples. In recent studies, overexpression of bcl2 was observed in lung cancer cells (Ma et al., 2019). In other study showed that $b c l 2$ expression and $b c l 2 /$ Bax expression level might be beneficial as independent diagnostic factors in lung carcinoids. Indeed, therapeutic approaches using down-regulation of $b c l 2$ might be useful in lung cancer (Velinovic et al., 2019). In a recent study, investigated sh-UCA1 significantly inhibited the protein levels of Beclin1 and LC3- and the cell growth and autophagy was decreased by down-regulated $L C 3$ expression (Yang et al., 2019). Although some studies show higher expression of $L C 3$ is correlated with higher growth for tumor cells, this indicated the autophagy markers $L C 3$ and $p 62$ are differentially expressed in NSCLC samples and high $L C 3$ expression seem to be linked to lower tumor aggressiveness, while high $p 62$ expression was significantly associated with aggressive tumor behavior (Schläfli et al., 2016). According to mentioned studies, LC3 may act as both oncogene and tumor suppressor gene in NSCLC. In this study, methylation of $b c l 2, L C 3$, ULK1 and Beclin1 were also analyzed and according to our data, $b c l 2$ gene was methylated in NSCLC samples compared to adjacent normal tissues, however, our data did not show a significant change about methylation of this gene. In recent studies, it has been indicated high expression levels of $p 53$ and $C H G A$ were correlated with the methylation of $b c l 2$ in NSCLC samples as well as methylation of $b c l 2$, $R A R B$ and SIX6 was associated with smoking. Moreover, they found methylation frequencies of 7 genes including bcl2 were significantly higher in stage I NSCLC than in non-cancerous lung diseases (Zhao et al., 2013). In another study demonstrated that bcl2 was hypo-methylated in NSCLC samples (Lokk et al., 2012). Indeed, methylation of this gene may have an essential impact on lung cancer progression. In this study, there was not any significant difference in the methylation pattern of LC3 in NSCLC 
patients compared to normal samples. However, some others indicated LC3Av1 was inactivated at the transcriptional level due to aberrant DNA methylation in esophageal squamous cell carcinoma (ESCC) cell lines and primary tumors. This result suggests that LC3Av1 functions may be pivotal in carcinogenesis (Bai et al., 2012). In our study, methylation of ULK1 was analyzed in tumoral samples compared with normal tissues and no significant difference was detected in NSCLC samples in comparison to normal tissues. In a study demonstrated that hyper-methylation of ULK2 promoter was detected by bisulfite sequencing in glioma cell lines. Indeed, ULK2 promoter methylation and transcript levels showed significant negative correlation. These data suggested that inhibition of autophagy via ULK1 down-regulation has a pivotal role for glioma development. Beclin1 was another factor which its methylation was analyzed in NSCLC compared to normal tissues. In our study, no methylation was occurred and expression pattern of this gene was increased significantly. Another study indicated TCF21 knockdown cells showed significantly upregulated $A T G-9$, Beclin1 and LC3-I/II expressions. Inhibition of autophagy by 3-methyladenine (3-MA) elevated TCF21 expression and increased cell apoptosis (Chen et al., 2018). Moreover, it has been analyzed miR-129-5p methylation and they found out hyper-methylation of this microRNA was associated with overexpression of Beclin1 in intervertebral disc degeneration (Zhao et al., 2017b).

In conclusion, $m i R-30 d$ and $m i R-204-5 p$ expression levels were decreased significantly and Beclin1, ULK1, bcl2 and $L C 3$ expression were significantly increased in NSCLC patients compared to normal adjacent tissues. These results suggesting that mentioned microRNAs might act as tumor suppressor genes, however, target genes might act as oncogenes in NSCLC samples. In addition, our data about methylation pattern of target genes did not show a significant change in tumoral samples comparing to adjacent normal tissues.

\section{Author Contribution Statement}

Study conception and design: Morteza Karimipoor, Shohreh Zare Karizi. Acquisition of data: Minoo Pargol, Shima Zare Karizi, Masoumeh Akbari, Mohammad Behgam Shadmehr. Analysis and interpretation of data:Minoo Pargol, Shima Zare Karizi, Masoumeh Akbar. Drafting of manuscript:Bahareh Nourmohammadi. Critical revision: Morteza Karimipoor, Shohreh Zare Karizi. The first three authors equally contributed to this work.

\section{Acknowledgments}

The authors sincerely thank all individuals who cooperated with this study.

\section{Ethical standards}

The study was approved by the ethics committee (sbmu 1. REC.1390. 65) of the Masih Daneshvari Hospital and written informed consent was taken from all patients before surgical resection.

Competing interests

The authors declare no conflict of interests.

\section{References}

Bai H, Inoue J, Kawano T, et al (2012). A transcriptional variant of the LC3A gene is involved in autophagy and frequently inactivated in human cancers. Oncogene, 31, 4397-408.

Bian Z, Jin L, Zhang J, et al (2016). LncRNA - UCA1 enhances cell proliferation and 5-fluorouracil resistance in colorectal cancer by inhibiting miR-204-5p. Sci Rep, 6, 23892.

Boolell V, Alamgeer M, Watkins DN, et al (2015). The evolution of therapies in non-small cell lung cancer. Cancers, 7, 1815-46.

Chen B, Zeng C, Ye Y, et al (2018). Promoter methylation of TCF21 may repress autophagy in the progression of lung cancer. J Cell Commun Signaling, 12, 423-32.

Chen Y, He J, Tian M, et al (2014). UNC51-like kinase 1, autophagic regulator and cancer therapeutic target. Cell Proliferation, 47, 494-505.

Chu Y, Jiang M, Du F, et al (2018). miR-204-5p suppresses hepatocellular cancer proliferation by regulating homeoprotein SIX 1 expression. FEBS Open Bio, 8, 189-200.

Frankel LB, Lund AH (2012). MicroRNA regulation of autophagy. Carcinogenesis, 33, 2018-25.

Frankel LB, Wen J, Lees M, et al (2011). microRNA-101 is a potent inhibitor of autophagy. EMBO J, 30, 4628-41.

Guo L, Zhao J, Qu Y, et al (2016). microRNA-20a inhibits autophagic process by targeting ATG7 and ATG16L1 and favors mycobacterial survival in macrophage cells. Front Cell Infect Microbiol, 6, 134.

Hannafon BN, Cai A, Calloway CL, et al (2019). miR-23b and miR-27b are oncogenic microRNAs in breast cancer: evidence from a CRISPR/Cas9 deletion study. BMC Cancer, 19, 642 .

He C, Klionsky DJ (2009). Regulation mechanisms and signaling pathways of autophagy. Annu Rev Genet, 43, 67-93.

Hou J, Aerts J, Den Hamer B, et al (2010). Gene expression-based classification of non-small cell lung carcinomas and survival prediction. PLoS One, 5.

Jian M, Yunjia Z, Zhiying D, et al (2019). Interleukin 7 receptor activates $\mathrm{PI} 3 \mathrm{~K} / \mathrm{Akt} / \mathrm{mTOR}$ signaling pathway via downregulation of Beclin-1 in lung cancer. $\mathrm{Mol}$ Carcinogenesis, 58, 358-65.

Jiang Z-F, Shao L-J, Wang W-M, et al (2012). Decreased expression of Beclin-1 and LC3 in human lung cancer. $\mathrm{Mol}$ Biol Rep, 39, 259-67.

Kondo Y, Kanzawa T, Sawaya R, et al (2005). The role of autophagy in cancer development and response to therapy. Nat Rev Cancer, 5, 726-34.

Lee HY, Shin JH, Lee K-Y, et al (2019). Prognostic role of beclin-1 in locally advanced non-small cell lung cancer in patients receiving docetaxel-platinum induction chemotherapy. Korean J Int Med, 34, 401.

Li S, Zeng X, Ma R, et al (2018). MicroRNA-21 promotes the proliferation, migration and invasion of non-small cell lung cancer A549 cells by regulating autophagy activity via AMPK/ULK1 signaling pathway. ExpTher Med, 16, 2038-45.

Liu Z, Huang S (2015). Inhibition of miR-191 contributes to radiation-resistance of two lung cancer cell lines by altering autophagy activity. Cancer Cell Int, 15, 16.

Lokk K, Vooder T, Kolde R, et al (2012). Methylation markers of early-stage non-small cell lung cancer. PLoS One, 7, e39813.

Luan W, Qian Y, Ni X, et al (2017). miR-204-5p acts as a tumor 
suppressor by targeting matrix metalloproteinases- 9 and B-cell lymphoma-2 in malignant melanoma. Onco Targets Ther, 10, 1237.

Ma G, Wang C, Lv B, et al (2019). Proteinase-activated receptor-2 enhances Bcl2-like protein-12 expression in lung cancer cells to suppress p53 expression. Arch Med Sci, 15, 1147.

Maiuri MC, Criollo A, Tasdemir E, et al (2007). BH3-only proteins and $\mathrm{BH} 3$ mimetics induce autophagy by competitively disrupting the interaction between Beclin 1 and Bcl-2/BclXL. Autophagy, 3, 374-6.

Miracco C, Cevenini G, Franchi A, et al (2010). Beclin 1 and LC3 autophagic gene expression in cutaneous melanocytic lesions. Hum Pathol, 41, 503-12.

Sacconi A, Biagioni F, Canu V, et al (2012). miR-204 targets Bcl-2 expression and enhances responsiveness of gastric cancer. Cell Death Dis, 3, e423.

Schläfli AM, Adams O, Galván JA, et al (2016). Prognostic value of the autophagy markers LC3 and p62/SQSTM1 in early-stage non-small cell lung cancer. Oncotarget, 7, 39544.

Sui X, Zhu J, Zhou J, et al (2015). Epigenetic modifications as regulatory elements of autophagy in cancer. Cancer Lett, 360, 106-13.

Sümbül AT, Göğebakan B, Ergün S, et al (2014). miR-204-5p expression in colorectal cancer: an autophagy-associated gene. Tumor Biol, 35, 12713-9.

Takahashi Y, Coppola D, Matsushita N, et al (2007). Bif-1 interacts with Beclin 1 through UVRAG and regulates autophagy and tumorigenesis. Nat Cell Biol, 9, 1142.

Tang F, Hu P, Yang Z, et al (2017). SBI0206965, a novel inhibitor of Ulk1, suppresses non-small cell lung cancer cell growth by modulating both autophagy and apoptosis pathways. Oncol Rep, 37, 3449-58.

Tang X, Jiang J, Zhu J, et al (2019). HOXA4-regulated miR-138 suppresses proliferation and gefitinib resistance in non-small cell lung cancer. Mol Genet Genomics, 294, 85-93.

Velinovic M, Jankovic R, Jovanovic D, et al (2019). Tumor characteristics, expressions of ERCC1, Bax, p53, IGF1R, $\mathrm{Bcl} 2, \mathrm{Bcl} 2 / \mathrm{Bax}$ and prognostic factors for overall survival in patients with lung carcinoid. $J B U O N, 24,256-66$.

Wang X, Du Z, Li L, et al (2015). Beclin 1 and p62 expression in non-small cell lung cancer: relation with malignant behaviors and clinical outcome. Int J Clin Exp Pathol, 8, 10644.

Xia Y, Zhu Y, Ma T, et al (2014). miR-204 functions as a tumor suppressor by regulating SIX1 in NSCLC. FEBS Lett, $\mathbf{5 8 8 ,}$ 3703-12.

$\mathrm{Xu}$ S, Pelisek J, Jin ZG (2018). Atherosclerosis is an epigenetic disease. Trends Endocrinol Metab, 29, 739-42.

Yang X, Zhong X, Tanyi JL, et al (2013). mir-30d Regulates multiple genes in the autophagy pathway and impairs autophagy process in human cancer cells. Biochemi Biophy Res Commun, 431, 617-22.

Yang Y, Liu X, Jin Z (2019). Effects of interference with UCA1 and inhibition of miR-185-5p on activation, autophagy and survival of $\beta$-Catenin pathway in non-small cell lung cancer. Sichuan da xue xue bao. Yi xue ban= J Sichuan Univ Med Sci Edition, 50, 157-63.

Yao J, Liang L, Huang S, et al (2010). MicroRNA-30d promotes tumor invasion and metastasis by targeting Galphai2 in hepatocellular carcinoma. Hepatology, 51, 846-56.

Zhao F, Qu Y, Wang H, et al (2017a). The effect of miR-30d on apoptosis and autophagy in cultured astrocytes under oxygen-glucose deprivation. Brain Res, 1671, 67-76.

Zhao K, Zhang Y, Kang L, et al (2017b). Methylation of microRNA-129-5P modulates nucleus pulposus cell autophagy by targeting Beclin-1 in intervertebral disc degeneration. Oncotarget, 8, 86264-76.
Zhao Y, Zhou H, Ma K, et al (2013). Abnormal methylation of seven genes and their associations with clinical characteristics in early stage non-small cell lung cancer. Oncol Lett, 5, 1211-8.

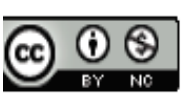

This work is licensed under a Creative Commons AttributionNon Commercial 4.0 International License. 